\title{
The genome sequence of the European water vole, Arvicola
}

\section{amphibius Linnaeus 1758 [version 1; peer review: 3 approved]}

\author{
Angus I. Carpenter (D1), Michelle Smith2, Craig Corton², Karen Oliver2, \\ Jason Skelton², Emma Betteridge2, Jale Doulcan (iD)2,3, Michael A. Quail2, \\ Shane A. McCarthy'2,4, Marcela Uliano Da Silva², Kerstin Howe (D2, \\ James Torrance (iD2, Jonathan Wood (D2), Sarah Pelan (iD2, Ying Sims2, \\ Francesca Floriana Tricomi (iD), Richard Challis (D)2, Jonathan Threlfall(D)2, \\ Daniel Mead (iD2,6, Mark Blaxter (iD)2

\footnotetext{
${ }^{1}$ Nottingham Trent University, Nottingham, NG1 4FQ, UK

${ }^{2}$ Wellcome Sanger Institute, Wellcome Genome Campus, Hinxton, Cambridge, CB10 1SA, UK

${ }^{3}$ Achilles Therapeutics PIc, London, W6 8PW, UK

${ }^{4}$ Department of Genetics, University of Cambridge, Cambridge, CB2 3EH, UK

${ }^{5}$ EMBL-EBI, Wellcome Genome Campus, Hinxton, Cambridgeshire, CB10 1SD, UK

${ }^{6}$ Owlstone Medical, Cambridge Science Park, Cambridge, CB4 0GJ, UK
}

V1 First published: 24 Jun 2021, 6:162

https://doi.org/10.12688/wellcomeopenres.16753.1

Latest published: 24 Jun 2021, 6:162

https://doi.org/10.12688/wellcomeopenres.16753.1

\section{Abstract}

We present a genome assembly from an individual male Arvicola amphibius (the European water vole; Chordata; Mammalia; Rodentia; Cricetidae). The genome sequence is 2.30 gigabases in span. The majority of the assembly is scaffolded into 18 chromosomal pseudomolecules, including the $X$ sex chromosome. Gene annotation of this assembly on Ensembl has identified 21,394 protein coding genes.

\section{Keywords}

Arvicola amphibius, European water vole, genome sequence, chromosomal

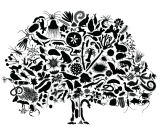

This article is included in the Tree of Life gateway.

\section{Open Peer Review}

\begin{tabular}{|c|c|c|c|}
\hline \multicolumn{4}{|c|}{ Approval Status } \\
\hline & 1 & 2 & 3 \\
\hline version 1 & $\checkmark$ & $\checkmark$ & $\checkmark$ \\
\hline 24 Jun 2021 & view & view & view \\
\hline
\end{tabular}

1. Petr Kotlik ID, Institute of Animal Physiology and Genetics of the Czech Academy of Sciences, Liběchov, Czech Republic

2. Alfonso Balmori-de la Puente (iD), Institute of Evolutionary Biology (CSIC-UPF),

Barcelona, Spain

Lídia Escoda ID, Institute of Evolutionary Biology (CSIC-UPF), Barcelona, Spain

3. Fahad Alqahtani (D), King Abdulaziz City for Science and Technology, Riyadh, Saudi Arabia Any reports and responses or comments on the 
article can be found at the end of the article.

Corresponding author: Mark Blaxter (mark.blaxter@sanger.ac.uk)

Author roles: Carpenter AI: Data Curation, Formal Analysis, Investigation, Resources, Writing - Review \& Editing; Smith M: Formal Analysis, Investigation, Methodology, Writing - Review \& Editing; Corton C: Formal Analysis, Investigation, Methodology, Writing Review \& Editing; Oliver K: Formal Analysis, Investigation, Methodology, Writing - Review \& Editing; Skelton J: Formal Analysis, Investigation, Methodology, Writing - Review \& Editing; Betteridge E: Formal Analysis, Investigation, Methodology, Writing - Review \& Editing; Doulcan J: Formal Analysis, Investigation, Methodology, Writing - Review \& Editing; Quail MA: Formal Analysis, Investigation, Methodology, Writing - Review \& Editing; McCarthy SA: Formal Analysis, Investigation, Methodology, Software, Validation, Writing Review \& Editing; Uliano Da Silva M: Formal Analysis, Investigation, Methodology, Software, Validation, Writing - Review \& Editing; Howe K: Formal Analysis, Investigation, Methodology, Software, Validation, Writing - Review \& Editing; Torrance J: Formal Analysis, Investigation, Methodology, Software, Validation, Writing - Review \& Editing; Wood J: Formal Analysis, Investigation, Methodology, Software, Validation, Writing - Review \& Editing; Pelan S: Formal Analysis, Investigation, Methodology, Software, Validation, Writing Review \& Editing; Sims Y: Formal Analysis, Methodology, Software, Visualization, Writing - Review \& Editing; Tricomi FF: Formal Analysis, Investigation, Methodology, Software, Validation, Writing - Review \& Editing; Challis R: Formal Analysis, Methodology, Software, Visualization, Writing - Review \& Editing; Threlfall J: Project Administration, Writing - Original Draft Preparation, Writing - Review \& Editing; Mead D: Conceptualization, Investigation, Project Administration, Writing - Review \& Editing; Blaxter M: Conceptualization, Data Curation, Funding Acquisition, Supervision, Writing - Review \& Editing

Competing interests: J. Threlfall was an employee of F1000Research up until January 2021.

Grant information: This work was supported by the Wellcome Trust through core funding to the Wellcome Sanger Institute (206194) and the Darwin Tree of Life Discretionary Award (218328).

The funders had no role in study design, data collection and analysis, decision to publish, or preparation of the manuscript.

Copyright: @ 2021 Carpenter AI et al. This is an open access article distributed under the terms of the Creative Commons Attribution License, which permits unrestricted use, distribution, and reproduction in any medium, provided the original work is properly cited.

How to cite this article: Carpenter AI, Smith M, Corton C et al. The genome sequence of the European water vole, Arvicola amphibius Linnaeus 1758 [version 1; peer review: 3 approved] Wellcome Open Research 2021, 6:162

https://doi.org/10.12688/wellcomeopenres.16753.1

First published: 24 Jun 2021, 6:162 https://doi.org/10.12688/wellcomeopenres.16753.1 


\section{Species taxonomy}

Eukaryota; Metazoa; Chordata; Craniata; Vertebrata; Euteleostomi; Mammalia; Eutheria; Euarchontoglires; Glires; Rodentia; Myomorpha; Muroidea; Cricetidae; Arvicolinae; Arvicola; Arvicola amphibius Linnaeus 1758 (NCBI:txid1047088).

\section{Introduction}

The European water vole, Arvicola amphibius Linnaeus 1758, is a small semi-aquatic mammal that lives on the banks of freshwater water courses and in wetlands. A. amphibius is native to Europe, west Asia, Russia and Kazakhstan. While the IUCN Red List of Threatened Species reports that A. amphibius is of "least concern" worldwide, populations in the United Kingdom have declined to such an extent that the species is considered nationally endangered (Mathews \& Harrower, 2020) owing to habitat loss and predation by the American mink, Neovison vison, an invasive alien species. An estimate by Natural England put the 2018 UK population of A. amphibius at 132,000, down from 7.3 million in 1990 (Strachan, 2004). Water voles are absent from Ireland. There have been a number of conservation projects in the UK aimed at supporting populations of A. amphibius, including efforts at habitat restoration and to control the population of American mink (Bryce et al., 2011). There are also efforts to reintroduce the water vole in a number of restored urban and wild habitats. This genome sequence will be of use as a reference for researchers that wish to assess the population genomics of A. amphibius and manage reintroductions.

\section{Genome sequence report}

The genome was sequenced from a single male A. amphibius collected from the Wildwood Trust, Herne Common, Kent, UK. A total of 45-fold coverage in Pacific Biosciences singlemolecule long reads (N50 $20 \mathrm{~kb}$ ) and 52-fold coverage in 10X Genomics read clouds (from molecules with an estimated N50 of $155 \mathrm{~kb}$ ) were generated. Primary assembly contigs were scaffolded with chromosome conformation Hi-C data. The final assembly has a total length of $2.298 \mathrm{~Gb}$ in 216 sequence scaffolds with a scaffold N50 of $138.7 \mathrm{Mb}$ (Table 1). The majority, $99.4 \%$, of the assembly sequence was assigned to 19 chromosomal-level scaffolds, representing 17 autosomes (numbered by sequence length apart from chromosome 12, which is larger because the previous version of the assembly, mArvAmp1.1, mistakenly labelled this as two separate chromosomes), and the X sex chromosome (Figure 1-Figure 4; Table 2). The assembly has a BUSCO (Simao et al., 2015) v5.0.0 completeness of $96.1 \%$ using the mammalia_odb10 reference set. While not fully phased, the assembly deposited is of one haplotype. Contigs corresponding to the second haplotype have also been deposited.

\section{Gene annotation}

The Ensembl gene annotation system (Aken et al., 2016) was used to generate annotation for an earlier version of the Arvicola amphibius assembly (GCA_903992535.1). Annotation was created primarily through alignment of transcriptomic data to the genome, with gap filling via protein to-genome

Table 1. Genome data for Arvicola amphibius, mArvAmp1.2.

\begin{tabular}{|l|l|}
\hline \multicolumn{2}{|l|}{ Project accession data } \\
\hline Assembly identifier & mArvAmp1.2 \\
\hline Species & Arvicola amphibius \\
\hline Specimen & mArvAmp1 \\
\hline NCBI taxonomy ID & txid1047088 \\
\hline BioProject & PRJEB39550 \\
\hline BioSample ID & SAMEA994740 \\
\hline Isolate information & Male; blood sample \\
\hline Raw data accessions & \\
\hline PacificBiosciences SEQUEL I & ERX3146757-ERX3146763 \\
\hline 10X Genomics Illumina & ERX3163119-ERX3163121, ERX3341539-ERX3341546 \\
\hline Hi-C Illumina & ERX3338011, ERX3338012 \\
\hline BioNano & ERZ1392829 \\
\hline Genome assembly & \\
\hline Assembly accession & GCA_903992535.2 \\
\hline Accession of alternate haplotype & GCA_903992525.1 \\
\hline Span (Mb) & 2,298 \\
\hline Number of contigs & 1,085 \\
\hline
\end{tabular}




\begin{tabular}{|c|c|}
\hline Contig N50 length (Mb) & 5.4 \\
\hline Number of scaffolds & 216 \\
\hline Scaffold N50 length (Mb) & 138.7 \\
\hline Longest scaffold (Mb) & 199.8 \\
\hline BUSCO* genome score & C:96.1\%[S:94.1\%,D:2.0\%],F:0.8\%,M:3.1\%,n:9226 \\
\hline \multicolumn{2}{|l|}{ Genome annotation } \\
\hline Number of protein-coding genes & 21,394 \\
\hline Average length of protein-coding gene (bp) & 1,700 \\
\hline Average number of exons per gene & 11 \\
\hline Average exon size (bp) & 208 \\
\hline Average intron size (bp) & 4,995 \\
\hline
\end{tabular}

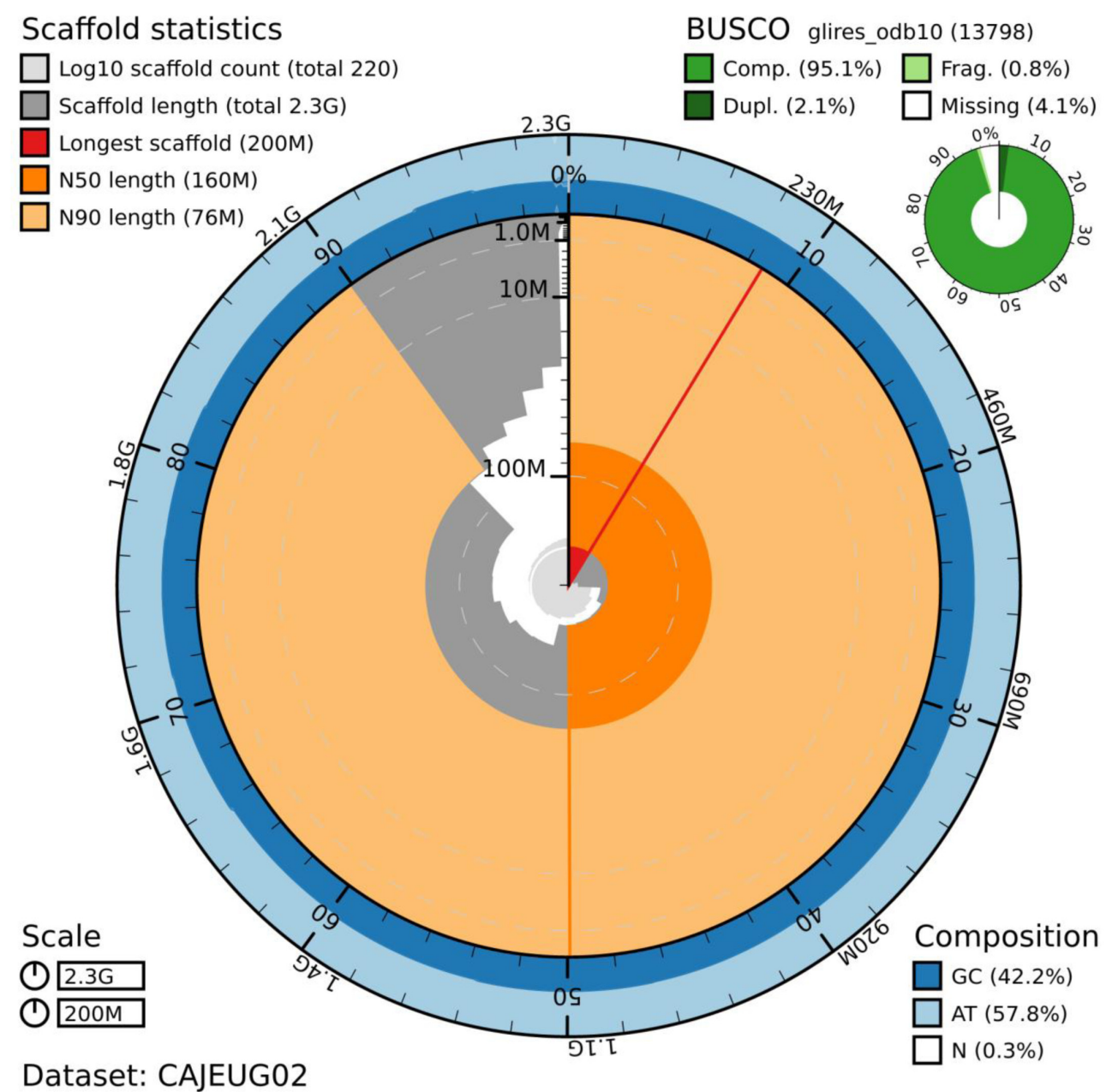

Figure 1. Genome assembly of Arvicola amphibius, mArvAmp1.2: metrics. The BlobToolKit Snailplot shows N50 metrics and BUSCO gene completeness. An interactive version of this figure is available at https://blobtoolkit.genomehubs.org/view/Arvicola\%20amphibius/ dataset/CAJEUG02/snail. 


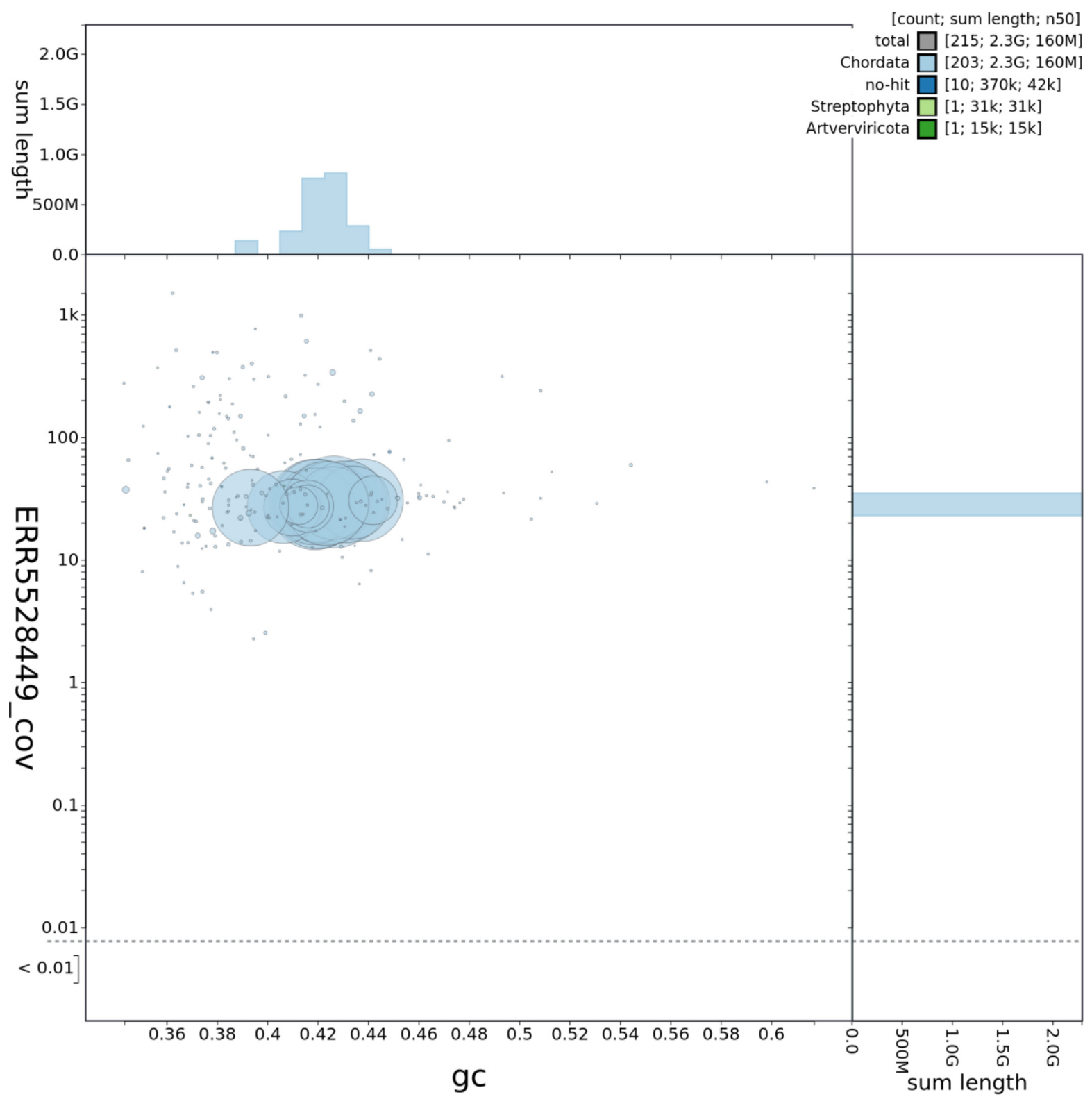

Figure 2. Genome assembly of Arvicola amphibius, mArvAmp1.2: GC coverage. BlobToolKit GC-coverage plot. An interactive version of this figure is available at https://blobtoolkit.genomehubs.org/view/Arvicola\%20amphibius/dataset/CAJEUG02/blob.

alignments of a select set of vertebrate proteins from UniProt (UniProt Consortium, 2019) and coordinate mapping of GENCODE (Frankish et al., 2019) mouse reference annotations via a pairwise whole genome alignment. The resulting Ensembl annotation includes 34,750 transcripts assigned to 21,394 coding and 2,252 non-coding genes (Arvicola amphibius - Ensembl Rapid Release).

\section{Methods}

A blood sample was taken from a live male A. amphibius specimen that was part of the captive breeding population of Wildwood Trust, Herne Common, Kent, UK (latitude 51.33181, longitude 1.11443). DNA was extracted using an agarose plug extraction from a blood sample following the Bionano Prep Animal Tissue DNA Isolation Soft Tissue Protocol. Pacific Biosciences CLR long read and 10X Genomics read cloud sequencing libraries were constructed according to the manufacturers' instructions. Sequencing was performed by the Scientific Operations DNA Pipelines at the Wellcome Sanger Institute on Pacific Biosciences SEQUEL I and Illumina HiSeq $\mathrm{X}$ instruments. Hi-C data were generated using the Dovetail v1.0 kit and sequenced on HiSeq X. Ultra-high molecular weight DNA was extracted using the Bionano Prep Animal Tissue DNA Isolation Soft Tissue Protocol and assessed by pulsed field gel and Qubit 2 fluorimetry. DNA was labeled for Bionano Genomics optical mapping following the 


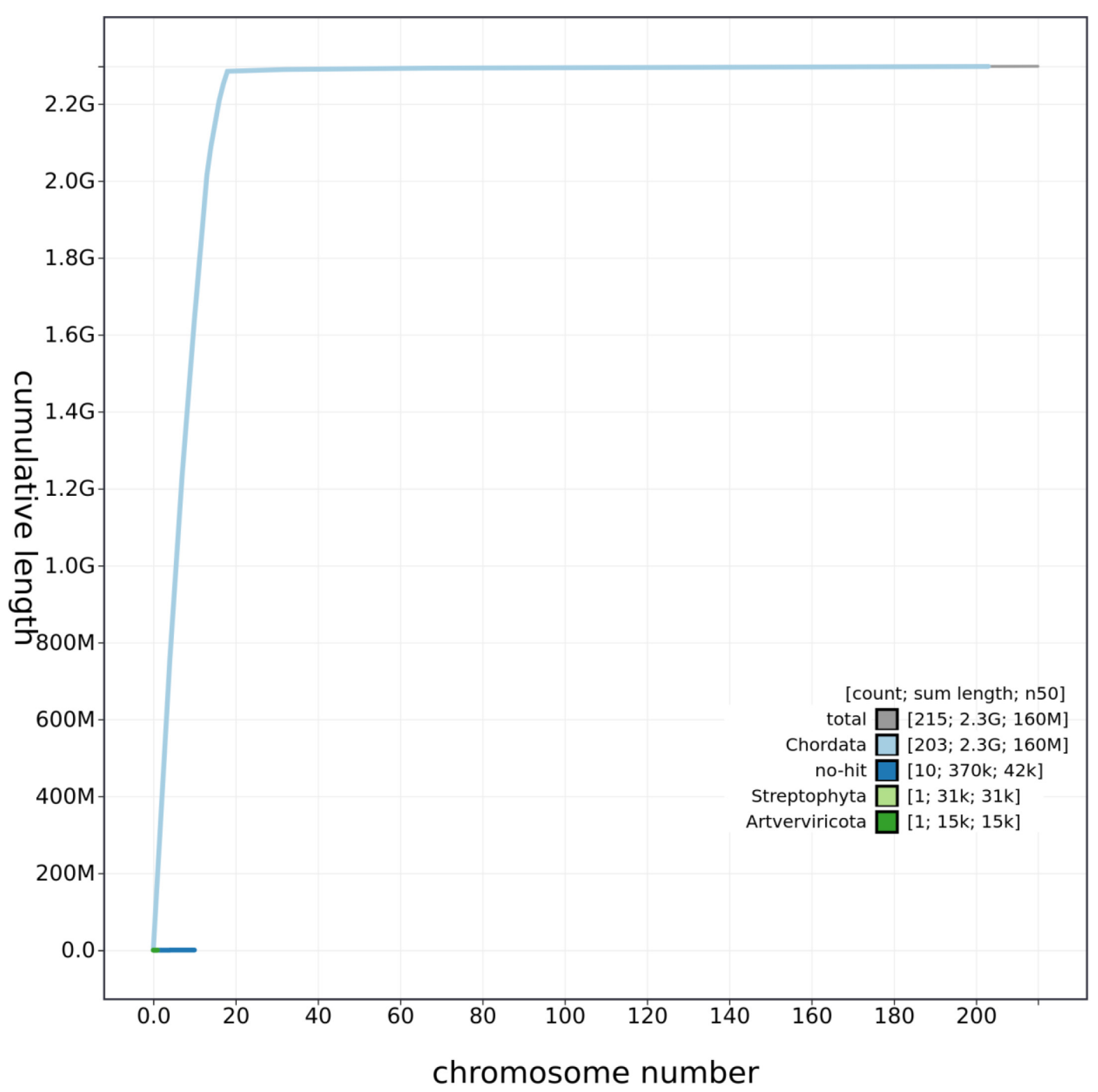

Figure 3. Genome assembly of Arvicola amphibius, mArvAmp1.2: cumulative sequence. BlobToolKit cumulative sequence plot. An interactive version of this figure is available at https://blobtoolkit.genomehubs.org/view/Arvicola\%20amphibius/dataset/CAJEUG02/ cumulative.

Bionano Prep Direct Label and Stain (DLS) Protocol and run on one Saphyr instrument chip flowcell.

Assembly was carried out following the Vertebrate Genome Project pipeline v1.6 (Rhie et al., 2020) with Falcon-unzip (Chin et al., 2016), haplotypic duplication was identified and removed with purge_dups (Guan et al., 2020) and a first round of scaffolding carried out with 10X Genomics read clouds using scaff10x. Hybrid scaffolding was performed using the BioNano DLE-1 data and BioNano Solve. Scaffolding with Hi-C data (Rao et al., 2014) was carried out with SALSA2 (Ghurye et al., 2019). The Hi-C scaffolded assembly was polished with arrow using the PacBio data, then polished with the 10X Genomics
Illumina data by aligning to the assembly with longranger align, calling variants with freebayes (Garrison \& Marth, 2012) and applying homozygous non-reference edits using bcftools consensus. Two rounds of the Illumina polishing were applied. The assembly was checked for contamination and corrected using the gEVAL system (Chow et al., 2016) as described previously (Howe et al., 2021). Manual curation was performed using evidence from Bionano (using the Bionano Access viewer), using HiGlass and Pretext, and by taking marker data and inspecting 10X barcode overlap using longranger. Figure 1-Figure 3 were generated using BlobToolKit (Challis et al., 2020). Table 3 contains a list of all software tool versions used, where appropriate. 


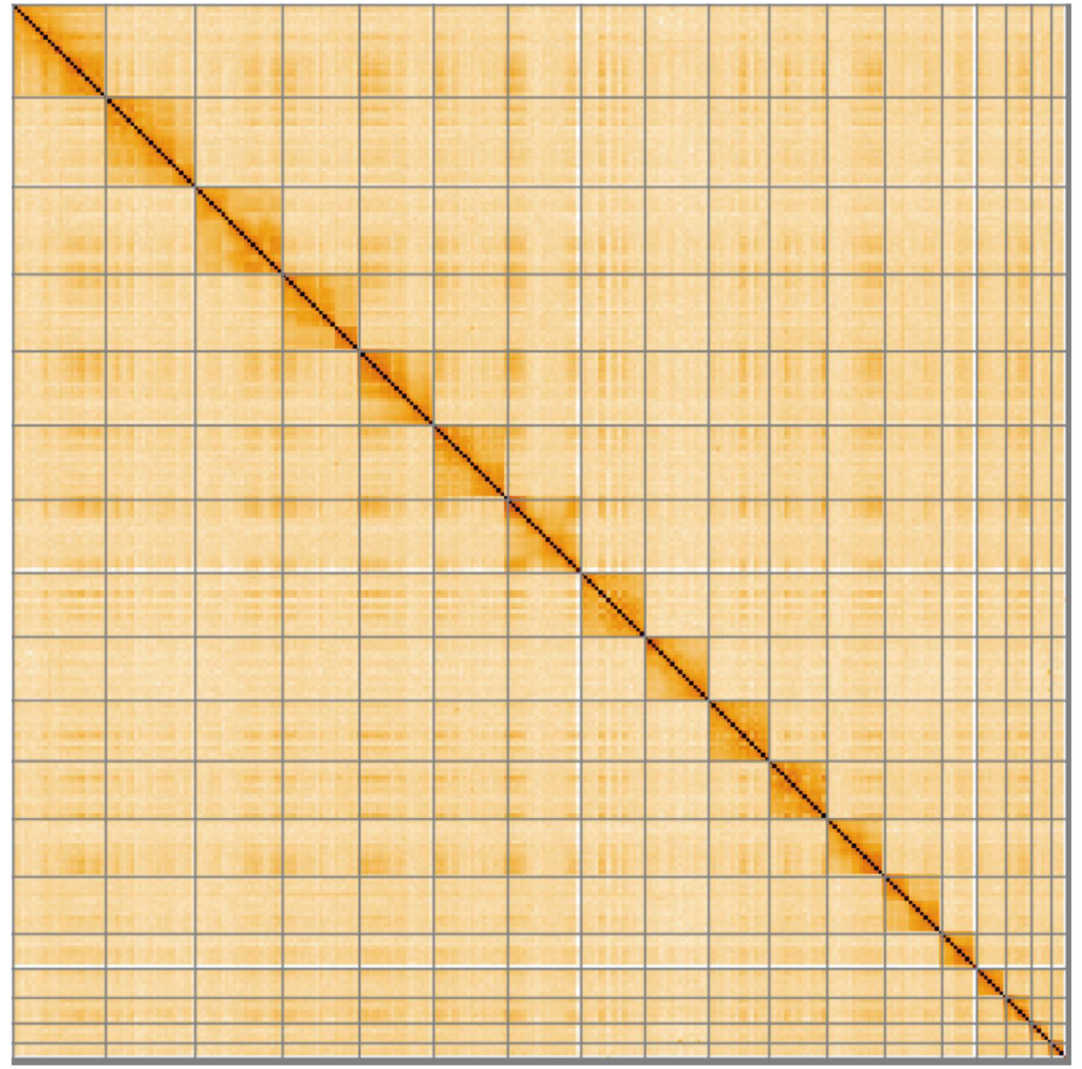

Figure 4. Genome assembly of Arvicola amphibius, mArvAmp1.2: Hi-C contact map. Hi-C contact map of the mArvAmp1 assembly, visualised in HiGlass.

Table 2. Chromosomal pseudomolecules in the genome assembly of Arvicola amphibius, mArvAmp1.2.

\begin{tabular}{|l|c|c|c|}
\hline INSDC accession & Chromosome & Size (Mb) & GC\% \\
\hline LR862380.1 & 1 & 200.53 & 42.7 \\
\hline LR862381.2 & 2 & 193.96 & 41.9 \\
\hline LR862382.1 & 3 & 189.60 & 42.0 \\
\hline LR862383.1 & 4 & 161.33 & 43.8 \\
\hline LR862384.1 & 5 & 160.72 & 42.6 \\
\hline LR862385.2 & 6 & 158.92 & 43.1 \\
\hline LR862386.1 & 7 & 138.66 & 41.9 \\
\hline LR862388.1 & 8 & 131.41 & 42.0 \\
\hline
\end{tabular}

\begin{tabular}{|l|c|c|c|}
\hline INSDC accession & Chromosome & Size (Mb) & GC\% \\
\hline LR862389.2 & 9 & 125.83 & 43.4 \\
\hline LR862390.1 & 10 & 125.09 & 42.6 \\
\hline LR862391.2 & 11 & 123.99 & 40.7 \\
\hline LR862392.2 & 12 & 166.75 & 42.3 \\
\hline LR862393.1 & 13 & 75.71 & 41.0 \\
\hline LR862394.1 & 14 & 63.16 & 41.6 \\
\hline LR862395.1 & 15 & 55.45 & 44.2 \\
\hline LR862397.2 & 17 & 42.65 & 41.6 \\
\hline LR862398.1 & 18 & 33.21 & 41.2 \\
\hline LR862387.1 & $X$ & 137.70 & 39.3 \\
\hline
\end{tabular}




\section{Table 3. Software tools used.}

\begin{tabular}{|c|c|c|}
\hline Software tool & Version & Source \\
\hline Falcon-unzip & falcon-kit 1.8 .0 & (Chin et al., 2016) \\
\hline purge_dups & $1.2 .3-b 542 \mathrm{dbf}$ & (Guan et al., 2020) \\
\hline SALSA2 & $2.2-14-g 974589 f$ & (Ghurye et al., 2019) \\
\hline scaff10x & 4.2 & https://github.com/wtsi-hpag/Scaff10X \\
\hline Bionano Solve & 3.3_10252018 & $N / A$ \\
\hline arrow & $\begin{array}{l}\text { gcpp 1.9.0-SL-release- } \\
8.0 .0+1-37-g d 7 b 188 d\end{array}$ & https://github.com/PacificBiosciences/GenomicConsensus \\
\hline longranger align & 2.2 .2 & $\begin{array}{l}\text { https://support.10xgenomics.com/genome-exome/software/ } \\
\text { pipelines/latest/advanced/other-pipelines }\end{array}$ \\
\hline freebayes & 1.3.1-17-gaa2ace8 & (Garrison \& Marth, 2012) \\
\hline bcftools consensus & $1.9-78-g b 7 e 4 b a 9$ & http://samtools.github.io/bcftools/bcftools.html \\
\hline gEVAL & N/A & (Chow et al., 2016) \\
\hline HiGlass & 1.11 .6 & (Kerpedjiev et al., 2018) \\
\hline PretextView & 0.0 .4 & https://github.com/wtsi-hpag/PretextMap \\
\hline BlobToolKit & 2.5 & (Challis et al., 2020) \\
\hline
\end{tabular}

\section{Data availability}

Underlying data

European Nucleotide Archive: Arvicola amphibius (European water vole) genome assembly, mArvAmp1. Accession number PRJEB39550.

The genome sequence is released openly for reuse. The Arvicola amphibius genome sequencing initiative is part of the Wellcome Sanger Institute's " 25 genomes for 25 years" project. It is also part of the Vertebrate Genome Project (VGP) ordinal references programme and the Darwin Tree of Life (DToL) project. All raw data and the assembly have been deposited in the ENA. The genome will be annotated and presented through the Ensembl pipeline at the European Bioinformatics Institute. Raw data and assembly accession identifiers are reported in Table 1.

\section{Acknowledgements}

We thank Mike Stratton and Julia Wilson for their continuing support for the 25 genomes for 25 years project.

\section{References}

Aken BL, Ayling S, Barrell D, et al.: The Ensembl Gene Annotation System. Database (Oxford). 2016; 2016: baw093.

PubMed Abstract | Publisher Full Text | Free Full Text

Bryce R, Oliver MK, Davies L, et al.: Turning Back the Tide of American Mink Invasion at an Unprecedented Scale through Community Participation and Adaptive Management. Biological Conservation. 2011; 144(1): 575-83. Publisher Full Text

Challis R, Richards E, Rajan J, et al: BlobToolKit - Interactive Quality Assessment of Genome Assemblies. G3 (Bethesda). 2020; 10(4): 1361-74. PubMed Abstract | Publisher Full Text | Free Full Text

Chin CS, Peluso P, Sedlazeck FJ, et al:: Phased Diploid Genome Assembly with Single-Molecule Real-Time Sequencing. Nat Methods. 2016; 13(12): 1050-54. PubMed Abstract | Publisher Full Text | Free Full Text

Chow W, Brugger K, Caccamo M, et al.: gEVAL - a Web-Based Browser for Evaluating Genome Assemblies. Bioinformatics. 2016; 32(16): 2508-10. PubMed Abstract | Publisher Full Text | Free Full Text

Frankish A, Diekhans M, Ferreira AM, et al.: GENCODE Reference Annotation for the Human and Mouse Genomes. Nucleic Acids Res. 2019; 47(D1): D766-73. PubMed Abstract | Publisher Full Text | Free Full Text

Garrison E, Marth G: Haplotype-Based Variant Detection from Short-Read Sequencing. arXiv:1207.3907. 2012

Reference Source

Ghurye J, Rhie A, Walenz BP, et al.: Integrating Hi-C Links with Assembly Graphs for Chromosome-Scale Assembly. PLoS Comput Biol. 2019; 15(8): e1007273.

PubMed Abstract | Publisher Full Text | Free Full Text

Guan D, McCarthy SA, Wood J, et al.: Identifying and Removing Haplotypic Duplication in Primary Genome Assemblies. Bioinformatics. 2020; 36(9): 2896-98.

PubMed Abstract | Publisher Full Text | Free Full Text

Howe K, Chow W, Collins J, et al.: Significantly Improving the Quality of Genome Assemblies through Curation. Gigascience. 2021; 10(1): giaa153. PubMed Abstract | Publisher Full Text | Free Full Text

Kerpedjiev P, Abdennur N, Lekschas F, et al.: HiGlass: Web-Based Visual 
Exploration and Analysis of Genome Interaction Maps. Genome Biol. 2018; 19(1): 125.

PubMed Abstract | Publisher Full Text | Free Full Text

Mathews F, Harrower C: IUCN - compliant Red List for Britain's Terrestria Mammals. Assessment by the Mammal Society under contract to Natural England, Natural Resources Wales and Scottish Natural Heritage. Natural

England, Peterborough, 2020

Reference Source

Rao SSP, Huntley MH, Durand NC, et al.: A 3D Map of the Human Genome at Kilobase Resolution Reveals Principles of Chromatin Looping. Cell. 2014;

159(7): 1665-80.

PubMed Abstract | Publisher Full Text | Free Full Text

Rhie A, McCarthy SA, Fedrigo O, et al.: Towards Complete and Error-Free
Genome Assemblies of All Vertebrate Species. bioRxiv. 2020;

2020.05.22.110833.

Publisher Full Text

Simão FA, Waterhouse RM, Ioannidis P, et al.: BUSCO: Assessing Genome Assembly and Annotation Completeness with Single-Copy Orthologs.

Bioinformatics. 2015; 31(19): 3210-12.

PubMed Abstract | Publisher Full Text

Strachan R: Conserving Water Voles: Britain's Fastest Declining Mammal. Water Environ J. 2004; 18(1): 1-4.

Publisher Full Text

UniProt Consortium: UniProt: A Worldwide Hub of Protein Knowledge.

Nucleic Acids Res. 2019; 47(D1): D506-15.

PubMed Abstract | Publisher Full Text | Free Full Text 


\section{Open Peer Review}

\section{Current Peer Review Status:}

\section{Version 1}

Reviewer Report 23 May 2022

https://doi.org/10.21956/wellcomeopenres.18475.r50219

(C) 2022 Alqahtani F. This is an open access peer review report distributed under the terms of the Creative Commons Attribution License, which permits unrestricted use, distribution, and reproduction in any medium, provided the original work is properly cited.

\section{Fahad Alqahtani}

National Center for Bioinformatics, King Abdulaziz City for Science and Technology, Riyadh, Saudi Arabia

This is an important study, and the authors have sequenced, assembled, and annotated a single male Arvicola amphibious (European water vole). According to the Wildlife Trusts, this species is endangered in Great Britain and on the England Red List for Mammals. From 1990 to 2018, the UK population of the European water vole dropped from 7.3 million to 132 hundred thousand. This population loses about $98 \%$ of its species in less than 30 years. Next-generation sequencing technologies are an efficient approach to generating life history and demographic data with respect to the management of endangered wildlife. Here, the authors have used different sequencing technologies (PacificBiosciences SEQUEL I, 10X Genomics Illumina, Hi-C Illumina, and BioNano) and generated a high-quality chromosomal-level assembly of this important species.

The manuscript is well written and well designed, and the results are clearly presented. The article adds much to the scientific community.

Is the rationale for creating the dataset(s) clearly described?

Yes

Are the protocols appropriate and is the work technically sound?

Yes

Are sufficient details of methods and materials provided to allow replication by others? Yes

Are the datasets clearly presented in a useable and accessible format? Yes

Competing Interests: No competing interests were disclosed. 
Reviewer Expertise: Applied algorithms in the bioinformatics field. Mitochondrial assembly and Mitochondrial haplogroup assignment.

I confirm that I have read this submission and believe that I have an appropriate level of expertise to confirm that it is of an acceptable scientific standard.

Reviewer Report 17 May 2022

https://doi.org/10.21956/wellcomeopenres.18475.r50221

(C) 2022 Balmori-de la Puente A et al. This is an open access peer review report distributed under the terms of the Creative Commons Attribution License, which permits unrestricted use, distribution, and reproduction in any medium, provided the original work is properly cited.

\author{
Alfonso Balmori-de la Puente \\ Institute of Evolutionary Biology (CSIC-UPF), Barcelona, Spain \\ Lídia Escoda \\ Institute of Evolutionary Biology (CSIC-UPF), Barcelona, Spain
}

This manuscript presents the annotated genome sequence of the European water vole (Arvicola amphibius), a small semiaquatic rodent distributed across Europe and Asia. Water vole populations of the genus Arvicola have a complex evolution with fossorial and semi-aquatic ecological types (ecotypes), thus this genome sequence can be very convenient to study ecological adaptations in rodents.

The report is well structured and clearly defined. However, there are some parts in the introduction that need to be better clarified.

The controversial taxonomic status of this genus, specifically between $A$. amphibius and its sister species $A$. scherman, and the complex genetic structure found in Great Britain is not properly assessed in the introduction. In addition, water voles of the genus Arvicola have broad ecological variability that should be better explained. Based on this, the species and the ecotype analyzed should be identified. In addition, the postglacial colonization events of water voles in the United Kingdom might be explained in more detail. All of these aspects will facilitate the future applications of the specimen sequenced for the conservation of the species.

Is the rationale for creating the dataset(s) clearly described?

Yes

Are the protocols appropriate and is the work technically sound?

Yes

Are sufficient details of methods and materials provided to allow replication by others? Yes

Are the datasets clearly presented in a useable and accessible format? 
Yes

Competing Interests: No competing interests were disclosed.

Reviewer Expertise: Conservation Genomics, Ecology and Evolution

We confirm that we have read this submission and believe that we have an appropriate level of expertise to confirm that it is of an acceptable scientific standard.

Reviewer Report 29 April 2022

https://doi.org/10.21956/wellcomeopenres.18475.r50220

(c) 2022 Kotlik P. This is an open access peer review report distributed under the terms of the Creative Commons Attribution License, which permits unrestricted use, distribution, and reproduction in any medium, provided the original work is properly cited.

\section{Petr Kotlik}

Laboratory of Molecular Ecology, Institute of Animal Physiology and Genetics of the Czech Academy of Sciences, Liběchov, Czech Republic

This is a short report presenting an annotated complete genome assembly for the water vole, a small mammal species that is widespread in continental Europe but declining and endangered in Britain. The high-quality genome presented here will be an important resource for studies addressing conservation genomics and other questions about the biology of the water vole.

The manuscript is clearly presented. I have not found any problems in it and therefore have no suggestions for changes.

Is the rationale for creating the dataset(s) clearly described?

Yes

Are the protocols appropriate and is the work technically sound? Yes

Are sufficient details of methods and materials provided to allow replication by others? Yes

Are the datasets clearly presented in a useable and accessible format?

Yes

Competing Interests: No competing interests were disclosed.

Reviewer Expertise: Evolutionary biology, population genomics, zoology, vertebrates

I confirm that I have read this submission and believe that I have an appropriate level of 
expertise to confirm that it is of an acceptable scientific standard. 